\title{
Analysis of Health Facility Based Barriers And Facilitators To Use Of Sexual And Reproductive Health Care Services Among Most At Risk Populations' (MARPS): Evidence From A Mystery Client Survey In Nigeria \\ Ezire $\mathrm{O}^{1 *}$, Okekearu $\mathrm{I}^{2}$, Fagbamigbe $\mathrm{A}^{3}$ and Faweya $\mathrm{O}$
}

${ }^{1}$ Research, Monitoring and Evaluation Advisor, Strengthening HIV Prevention Services for Most at Risk Population, Abuja, Nigeria

${ }^{2}$ Society for Family Health, Abuja

${ }^{3}$ University of Ibadan, Nigeria

${ }^{4}$ Prevention Advisor, Strengthening HIV Prevention Services for Most at Risk Population, Abuja, Nigeria

${ }^{5}$ Department of Epidemiology and Medical Statistics, College of Medicine, University of Ibadan, Nigeria.

\begin{abstract}
Background: Access to sexual and reproductive health care services for most at risk population (MARPs) is a very important step in stemming the spread of HIV; as HCT and early diagnosis and treatment of STIs can contribute to reduction of the spread of HIV. Within Nigeria and most Sub Saharan African countries, MARPs are highly stigmatized both in the general society and in health care centres. It is important health care facility based facilitators and barriers to MARPs' access to use of sexual and reproductive health care services by MARPs are identified to guide project design and implementation as well as policy formulation.
\end{abstract}

Methods: Mystery client survey was conducted in thirty three (33) healthcare facilities in Nigeria. For the purpose of the survey, the mystery clients (MARPs) sought only STI consultation and HIV counselling and testing services. Twelve (12) mystery clients visited each facility seeking either STI or HCT service in the facility. Ethical approval was obtained from the Nigeria Institute of Medical Research (NIMR). SPSS version 20 was used for the Bi-variate and multivariate logistic regression analysis.

Results: While positive attitude of health care workers was found to be a facilitator of access to health care services, this study shows that perceived quality of services is a very significant variable in MARPs' use of a health care facility. We also found that MARPs were more willing to refer their community members to facilities that have educational materials promoting non-stigmatizing attitude towards PLWHAs and MARPs. Amazingly, privacy of services provided was not significant.

Conclusion: It is recommended that standard of health care practices should be enforced. Training and re training of health care workers on provision of MARP friendly services should be formalized and scaled up. Investment in educational materials (posters) in health facility settings should be promoted.

Keywords: Quality health care services; Attitude of health care workers; MARPs; Training of health care workers; Confidentiality and privacy

\section{Introduction}

In Nigeria, HIV prevalence among general population is estimated to be 3.2 percent [1] this equates to around 3.4 million people living with HIV putting it only second behind South Africa in terms of absolute numbers [2]. In most Sub Saharan Countries Nigeria inclusive, HIV prevalence among most at risk population (MARPs) is higher than what is found in the general population [3]. For example in Nigeria, while HIV prevalence is above $20 \%$ and $15 \%$ among female sex workers and men who have sex with men respectively, prevalence among general population is 3.2 percent $[1,4]$. Communities most affected by the epidemic-sex workers, Injecting Drug Users (IDUs), men who have sex with men (MSM) and transgender people remain highly stigmatized; these individuals and their families are often unable to exercise their rights to health, non-discrimination and freedom from violence [5]. There is no question that the stigma and discrimination associated with HIV and AIDS can be reduced through stigma reduction intervention. The inclusion of stigma and discrimination reduction as a critical component of achieving an AIDS-free generation in recent UNAIDS, UN and PEPFAR political initiatives is promising [5]. Consistent data highlights the central role of stigma in limiting uptake of HIV prevention, treatment and care services [6,7]. This is especially true among MARPs including men who have sex with men
(MSM), who are at elevated risk of HIV acquisition and transmission, live outside of broad social expectations for gender roles, and therefore often experience homoprejudice [8]. Studies from Malawi, Botswana and Namibia have demonstrated that MSM who had any interaction with healthcare had over two times greater odds of experiencing fear of seeking healthcare and over six times greater odds of having been denied healthcare due to sexual orientation [9]. Moreover, studies in other Sub-Saharan African countries have found disclosure of one's sexual orientation (homosexual or sex worker) to healthcare workers or family members associated with nearly four times and nearly three times increased odds of blackmail respectively [10]. In qualitative research in South Africa, MARPs reported verbal discrimination by healthcare workers who know them. This resulted in non-disclosure of their HIV status by bisexually identified MSM. They also resort to

*Corresponding author: Onoriode Ezire, Research, Monitoring and Evaluation Advisor, Strengthening HIV Prevention Services for Most at Risk Population, Abuja, Nigeria, Tel: +2348060087524; E-mail: oeziree@yahoo.com

Received July 16, 2015, 2015; Accepted August 12, 2015; Published August 19, 2015

Citation: Ezire O, Okekearu I, Fagbamigbe A, Faweya O (2015) Analysis of Health Facility Based Barriers And Facilitators To Use Of Sexual And Reproductive Health Care Services Among Most At Risk Populations' (MARPS): Evidence From A Mystery Client Survey In Nigeria. J Trop Dis 3:171. doi:10.4172/2329891X.1000171 Copyright: (C) 2015 Ezire O, et al. This is an open-access article distributed unde the terms of the Creative Commons Attribution License, which permits unrestricted use, distribution, and reproduction in any medium, provided the original author and source are credited. 
Citation: Ezire O, Okekearu I, Fagbamigbe A, Faweya O (2015) Analysis of Health Facility Based Barriers And Facilitators To Use Of Sexual And Reproductive Health Care Services Among Most At Risk Populations' (MARPS): Evidence From A Mystery Client Survey In Nigeria. J Trop Dis 3:171. doi:10.4172/2329891X.1000171

travelling long distances to seek appropriate care [11,12]. Health care workers have been identified in several studies as barriers to access to services [13]. Healthcare workers (HCWs) in Africa typically receive little or no training in the healthcare needs of Most At Risk Population including men who have sex with men (MSM), limiting the effectiveness and reach of population-based HIV control measures among this group [14]. A study in Coastal Kenya concluded that scaling up MSM sensitivity training for African Health Care Workers (HCW) is likely to be a timely, effective and practical means to improve relevant sexual health knowledge and reduce personal homophobic sentiments among HCWs involved in HIV prevention, testing and care in sub-Saharan Africa [14].

\section{Rationale for the study}

In Nigeria, HIV prevalence among MARPS is far higher than what is found among general population (FSWs: $22.0 \%$; MSM: $17.9 \%$ and IDUs: $4.9 \%$ ) [4]. Improved access to HCT and STI services is crucial if the epidemic is to be curtailed especially among MARPs [15]. Access to sexual and reproductive health care services by MARPs is likely to improve if they are satisfied with services received. This will reflect in their willingness to revisit the facility and most importantly refer members of their community to the facility for similar services. While some efforts has been made to build capacity of health care providers to offer non stigmatized health care services to most at risk population (MARPs), very limited evidence exists that identifies other possible factors that influence MARPs to seek and or to continue to seek health care services in a particular health facility and also importantly, to refer their community members to the facility.

\section{Research questions}

1. What factors motivate MARPs to refer their community members for either HCT or STI consultation services in a health facility?

2. Which of these factor(s) is (are) most significant in influencing MARPs to seek health care services in a health facility?

\section{Operational definitions}

\begin{tabular}{|c|c|}
\hline $\begin{array}{c}\text { Female Sex } \\
\text { Workers (FSWs) }\end{array}$ & $\begin{array}{c}\text { Any female aged 15 years and above, who receives } \\
\text { money or other valuable gifts/incentives in exchange } \\
\text { for sex in areas such as brothels, bars, restaurants, } \\
\text { nightclubs, hotels, or on the street [4]. Which within Nigeria } \\
\text { context FSW can be as young as 15, in this study, only } \\
\text { those 18 years and above participated. }\end{array}$ \\
\hline $\begin{array}{c}\text { Men who have sex } \\
\text { with men (MSM) }\end{array}$ & $\begin{array}{c}\text { A male 18 years and above who has engaged in an anal } \\
\text { sexual activity with other man or men in the last 12 months } \\
\text { preceding the survey [4]. }\end{array}$ \\
\hline $\begin{array}{c}\text { Injecting Drug Users } \\
\text { (IDUs) }\end{array}$ & $\begin{array}{c}\text { Any male or female aged 18 years and above who has } \\
\text { injected psychoactive drugs recreationally at least once in } \\
\text { the past 12 month [4]. }\end{array}$ \\
\hline $\begin{array}{c}\text { HIV Counseling } \\
\text { and Testing (HCT) } \\
\text { Services }\end{array}$ & $\begin{array}{c}\text { This covers pre and post HIV test counseling and actual } \\
\text { HIV test services. Within the context of this study, services } \\
\text { are only completed if the three components are covered. }\end{array}$ \\
\hline $\begin{array}{c}\text { Sexually Transmitted } \\
\text { Infection (STI) } \\
\text { Consultation } \\
\text { Services }\end{array}$ & $\begin{array}{c}\text { This covers STI counseling and consultation services } \\
\text { provided by a qualified health care worker. It excludes } \\
\text { actual treatment of STls. }\end{array}$ \\
\hline
\end{tabular}

\section{Study Methodology}

Data for the study was collected through a mystery client survey with MARPs as the clients [16]. The survey was conducted in 33 health facilities purposefully selected. The facilities were selected, as they are referral centres, for the Strengthening HIV Prevention Services for MARPs project. Ethical approval was obtained from Nigeria Institute of
Medical Research (NIMR) with IRB number 12201.

\section{Selection and training of mystery clients}

Mystery clients were selected from a pool of MARPs the Strengthening HIV Prevention Services for MARPs project in Nigeria is working with. Participation was voluntary. In the selection of mystery clients, the following were used as inclusion criteria: the person must be a MARP (FSW, MSM or IDU); must be at least 18 years old and must have been in the community for at least one year. For the HCT component, the clients recruited were MARPs who had been tested before and are aware of their HIV status. Through personal discussions, willing mystery clients were psychologically prepared to go through the HCT process again. To affirm their preparedness, all mystery clients were asked to sign a release form. Twelve (12) mystery clients were recruited (4 FSWs; 4 MSM, 4 IDUs), six to seek STI services while the other six sought HCT services in each facility. The mystery clients were taken through a two-day training session where the purpose of the study was made known to them. Mock sessions were held, observations were noted and corrections made where necessary.

\section{Data collection}

Data was collected using a structured observation checklist, which was pretested for consistency and validity before actual fieldwork. The following steps were followed by each mystery client in the course of completing the checklist: mystery client visited an assigned facility using the assigned scenario (STI consultation services or HCT services), made notes immediately after the visit, complete the checklist and discussed the outcome of the visit with the supervisor immediately after leaving the facility to aid recall of event.

\section{Data analysis}

Data were analyzed using SPSS version 20. Bivariate analyses were conducted to determine if the Independent variables were significantly associated with the outcome variable using chi square. Independent variables that were significantly associated with the dependent variable (bivariate analysis) were included in a multiple logistic regression to determine the effect of each independent variable after controlling for others. Significance was tested at $5 \%$ level of significance.

\section{Variables in the equation}

Dependent variables: Willingness to refer a community member to the facility for HCT services or not. This is coded "I" for yes, and " 0 " for no. For STI consultation services, willingness to refer community member to the facility for similar service is coded "I" for yes, and "0" for no.

\section{Independent Variables were the following}

Ownership: This variable is used to capture the ownership and management of the health facility. If publicly owned and managed, it is coded " 1 ", if not it is coded " 0 ".

Location: Location within the context of this study, location refers to North or Southern Nigeria with South coded "1" and North, "0".

Training: This is used to capture whether staff in the facility have been trained on the provision of MARP friendly services. If a staff in the facility has been trained on provision of MARP friendly services, it is coded " 1 "; if not " 0 ".

Promo materials: This variable was computed from several questions on IEC materials in the facility. There were three possible responses (sufficiently: 3; barely: 2 and none: 1). The areas assessed 
Citation: Ezire O, Okekearu I, Fagbamigbe A, Faweya O (2015) Analysis of Health Facility Based Barriers And Facilitators To Use Of Sexual And Reproductive Health Care Services Among Most At Risk Populations' (MARPS): Evidence From A Mystery Client Survey In Nigeria. J Trop Dis 3:171. doi:10.4172/2329891X.1000171

were whether displayed or available IEC materials:

- $\quad$ Made reference to HIV prevention

- $\quad$ Made reference to voluntary counselling and testing for HIV

- Have messages inclusive of sexually diverse populations and their needs

- Have messages addressing stigma and discrimination against people living with HIV or other most-at-risk populations

- Made positive references to the range of gender identities, sexual orientations or occupations; materials at the facility appropriate for MARPs needs

- $\quad$ Captured the rights and perspective of MARPs.

Propensity scoring was done to have one variable (promotional materials) and three categories of responses were computed: poor, barely sufficient and sufficient. In the multiple logistic regression analysis, this was re-coded as: Sufficiently: "1"; Not sufficiently (either Poor or barely): " 0 ".

Privacy and confidentiality: The following items/variables were used to compute privacy and confidentiality using propensity scoring:

- MARP counselled in a separate room: yes "2", no "1";

- MARP counselled in a place where other persons can't hear the conversation: yes " 2 ", no " 1 ";

- any interruptions during counselling session/consultation (e.g. health care worker answered phone, someone else walked into speak with health care worker): no: " 3 "; yes once " 2 "; yes several times "1";

- health care worker assured MARP of the confidentiality of the counselling session: yes "2", no "1";

- Health care worker offered MARP to use a number, pseudonym or another name instead of real name to ensure confidentiality: yes " 2 ", no " 1 "

- Registers of other clients' information kept out of sight during the counselling session / consultation / testing: yes " 2 ", no " 1 ".

Responses were summed up and re-classed as: poor, barely and sufficiently. In the logistic regression analysis, privacy and confidentiality was re-coded as " 1 " if adequately confidential; and " 0 " if not.

Health care workers' attitude: This was computed from three variables using propensity scoring:

- Health care worker was friendly and welcoming: yes: "2" and no: "1";

- Health care worker exhibited a non-judgmental attitude: no: " 3 "; yes, somewhat " 2 " and yes completely " 1 " and

- Health care worker was supportive: yes completely " 3 "; yes somewhat "2" and no "1".

Responses were categorized as: poor, fair or acceptable. These were re-classified as: sufficiently acceptable "1" and not sufficiently acceptable (poor and fair) "0" in the logistic regression analysis.

Quality of HCT and STI services: Based on the national guidelines for the provision of HCT and STI services, responses were categorized into: very poor, poor, fair, good and excellent. In the logistic regression, quality of services was re-coded " 1 " if quality of service adequately meets minimum recommended standard (good or excellent) and " 0 " if not (fair, poor or very poor). Variables used are listed in the appendix.

\section{Results}

\section{Respondents' profile}

Three hundred and forty three questionnaires were completed as against the expected three hundred and ninety three. 16 were not included in the analysis as the type of client code was not properly filled. Of the valid responses, FSWs account for $32.4 \%$, IDUs: $32.4 \%$ and MSM: $35.2 \%$. In terms of service sought, $57.1 \%$ sought HIV counseling and testing services while $42.9 \%$ sought STI consultation service. $44.9 \%$ sought services in a private facility, $52.5 \%$ in a facility located in southern Nigeria and $33.2 \%$ in facilities where staff has been trained on MARP friendly services.

\section{Bivariate analysis}

HCT services: The significance of the independent variables to the outcome variable was tested using chi-square test. There appears to be no significant difference between respondents who visited private and public facilities ( $p$ : 0.541 ), respondents who visited facilities located in the south and north ( $p$ : 0.173) and those who feel services were privately and confidentially provided or not ( $p$ : 0.494). Training of health workers, adequate promotional materials in the facility; attitude of health care workers and quality of services were very significant variables $(p: 0.004 ; 0.001 ; 0.0001$ and 0.0001 respectively).

STI consultation services: Type of ownership (public or private) ( $p$ : $<0.001)$, training $(p: 0.017)$, promotional materials $(p:<0.001)$ and quality of services ( $p: 0.002)$ were significant as $5 \%$ level of significance. Location of the facility (south or north) ( $p$ : 0.311); services provided privately and confidentially ( $p: 0.067)$ and attitude of health care workers ( $p$ : 0.379) were not significant. Details are presented in Table 1.

\section{Multiple logistic regressions}

The strength of the association between the dependent and the independent variables was tested using multiple logistic regressions. Only variables that were significant in the bivariate analysis were included in the analysis.

HCT services: MARPs who feel quality of HCT services meets minimum required as recommended in the national HCT guideline are over $300 \%$ most likely to recommend a community member to the facility as compared with those who feel the services do not meet required minimum standard (p: 0.036; OR: 4.012 and CI: 1.096 and 14.688). Those who perceived attitude of health workers to be acceptable are $23 \%$ less likely to refer their community member to the facility as compared with those who feel otherwise (p: 0.001; OR: 0.023 and CI: 0.002 and 0.223 ). Training of staff and promotional materials were not significant at $5 \%$ level of significant.

STI Consultation services: Training of staff, promo materials and type of ownership were significant variables that explained variations in the dependent variable at 5\% level of significance. MARPS are almost $300 \%$ more likely to refer community members to a facility for STI services where staff are trained as compared with facilities where staff were not trained ( $p$ : 0.035; OR: 3.460 and CI: 1.090 and 10.985); almost 700\% more likely to refer to the facility if there are adequate promo materials compared with facilities without adequate promo materials ( $p$ : 0.002 ; OR: 7.667; CI: 2.140 and 27.474$)$ and $18 \%$ less likely if the facility is publicly owned as compared with a private facility ( $p$ : 0.001 ; OR: 0.180 CI: 0.064 and 0.505$)$. Quality of services was not significant at $5 \%$ level 
Citation: Ezire O, Okekearu I, Fagbamigbe A, Faweya O (2015) Analysis of Health Facility Based Barriers And Facilitators To Use Of Sexual And Reproductive Health Care Services Among Most At Risk Populations' (MARPS): Evidence From A Mystery Client Survey In Nigeria. J Trop Dis 3:171. doi:10.4172/2329891X.1000171

\begin{tabular}{|c|c|c|c|c|c|c|}
\hline \multirow[b]{2}{*}{ Variables } & \multicolumn{3}{|c|}{ HCT services } & \multicolumn{3}{|c|}{ STI consultation services } \\
\hline & $\mathbf{n}$ & $\%$ & p-value & $\mathbf{n}$ & $\%$ & p-value \\
\hline Publicly Owned & 198 & 71.6 & 0.541 & 143 & 48.1 & 0.0001 \\
\hline Southern Located & 198 & 67.7 & 0.173 & 143 & 61.2 & 0.311 \\
\hline Staff in facility trained & 198 & 83.1 & 0.004 & 143 & 78.0 & 0.017 \\
\hline Promo Materials adequate & 178 & 86.2 & 0.001 & 125 & 89.2 & 0.0001 \\
\hline Services are offered privately and confidentially & 173 & 75.8 & 0.494 & 135 & 81.0 & 0.067 \\
\hline HCW Attitude is acceptable & 193 & 20.8 & 0.0001 & 137 & 65.4 & 0.379 \\
\hline Quality of service meet minimum requirements & 124 & 91.5 & 0.001 & 110 & 73.1 & 0.002 \\
\hline
\end{tabular}

Table 1: Result of Bivariate analysis.

\begin{tabular}{|c|c|c|c|c|c|c|c|c|}
\hline & \multicolumn{4}{|c|}{ HCT Services } & \multicolumn{4}{|c|}{ STI consultation Services } \\
\hline & \multirow{2}{*}{$p$-value } & \multirow{2}{*}{ OR } & \multicolumn{2}{|c|}{$95 \%$ C.I. for OR } & \multirow{2}{*}{$p$-value } & \multirow{2}{*}{ OR } & \multicolumn{2}{|c|}{ 95\% C.I. for OR } \\
\hline & & & Lower & Upper & & & Lower & Upper \\
\hline HCW Attitude is acceptable & .001 & .023 & .002 & .223 & & & & \\
\hline Staff in facility trained & .300 & 1.988 & .542 & 7.295 & .035 & 3.460 & 1.090 & 10.985 \\
\hline Promo Materials adequate & .275 & 2.040 & .567 & 7.338 & .002 & 7.667 & 2.140 & 27.474 \\
\hline $\begin{array}{l}\text { Quality of service meet } \\
\text { minimum requirements }\end{array}$ & .036 & 4.012 & 1.096 & 14.688 & .081 & 2.337 & .901 & 6.064 \\
\hline Publicly Owned & & & & & .001 & .180 & .064 & .505 \\
\hline Constant & .101 & 1.786 & & & .570 & 1.284 & & \\
\hline
\end{tabular}

Table 2: Result of multiple logistic regressions.

of significance ( $p$ : 0.081). Details are presented in Table 2.

\section{Discussions}

This study attempted to look at the various possible facility based facilitators and barriers to access to health care services from the view point of MARPs seeking health care services (HIV Counseling and Testing services and STI consultation services). For HCT services: ownership of the facility (privately or publicly owned), location of the facility (either in Northern or Southern part of Nigeria) and confidentiality and privacy of the service provided were not significant factors that will make MARPs to further access services in the facility or refer their community member to access HCT services in the facility. On the other hand, quality of services provided (defined as meeting the minimum standard set in the guidelines for provision of HCT services [17], non-judgmental attitude of health care workers and presence of educational materials promoting HCT services are very significant variables that promote use of health facilities by MARPs. The study also revealed that MARPs are four times more likely to access HCT services in facilities where minimum HCT standards are met as compared to where there are not. For STI consultation services, attitude of health care workers was not significant whereas quality of services provided, training of health care workers on provision of MARPs friendly services and existence of educational materials in the facility were the significant variables. This study has shown that while attitude of health care workers is significant in promoting access to health care services on the part of MARPs, quality of services being provided is also very important. The presence of educational materials on sexuality and rights possibly gives the impression that you are welcome irrespective of who you are; this non-verbal communication communicates acceptance. Surprisingly, privacy of the services provided was not significant. This may be explained by the fact that the crowded nature of most of our health care centres do not guarantee secrecy and so most people have associated non-privacy of services as the common norm. This study however shows that location of practice is not a very significant variable that explains access to health care services by MARPs. It is possible that the results would have been different if the analysis were done according to categories of MARPs. Unfortunately, we could not because of the sample size. It is also possible that if actual STI services were provided, we would have had different results. The study team was not with the clients when accessing services. We only rely on the training that they will disclose who they are to the health care worker. To what extent this was done was not assessed. Actual disclosure of who they are can significantly change the outcome of the study.

\section{Conclusion}

This study corroborates some other studies and opinions that when health care workers have access to complete and accurate information, patients receive better medical care $[18,19]$. It also shows that when minimum service provisions are met, MARPs will access services in the facility. The study also corroborates other studies on the importance of attitude of health care workers in promoting access to health care services $[9,19]$. When health workers demonstrate non-judgmental attitude, access to health care services is promoted in those facilities. We relied on the ability of the respondent to recall and properly fill the questionnaire. Recall bias may have some effect on the results [16]. It brought some new evidences that presence of educational materials in a health care facility (that promotes equal access to health, educational information of HIV, STI, PLWHA etc.) are very subtle ways to communicate to MARPs that they are welcome. Training of HCWs on MARPs friendly services can contribute to promoting access to health care services by MARPs [19]. This is very important if we are to stem the tide of HIV infection in the country. It is recommended that a standard training curriculum on provision of MARPs friendly services is developed and taught in medical schools. Setting minimum standards is a common practice in health care service. The norm in Nigeria is that very little attention is paid to their enforcement. This study has shown that setting standards does work. MARPs and by extension other health care users are most likely to keep visiting centres that offer quality services. Government agencies responsible for enforcing standards need to be more active and ensure that quality standards are met by health care workers. Very little is known on the link between posters / educational materials in health care centres and the impact on access to health care services. This study has shown some positive relationship. Investment in these promo materials are worth it and 
Citation: Ezire O, Okekearu I, Fagbamigbe A, Faweya O (2015) Analysis of Health Facility Based Barriers And Facilitators To Use Of Sexual And Reproductive Health Care Services Among Most At Risk Populations' (MARPS): Evidence From A Mystery Client Survey In Nigeria. J Trop Dis 3:171. doi:10.4172/2329891X.1000171

should be promoted. The study did not show any relationship between privacy and confidentiality and access to health care services. It is not clear why we had this result. Further research on this is recommended.

\section{Conflict of Interest}

We have no conflict of interest

\section{Acknowledgements}

All MARPS that voluntarily participated in the survey. We are also grateful to the health care facilities used for the study.

\section{References}

1. Federal Ministry of Health (2010) Nigeria National HIV Seroprevalence survey among pregnant women.

2. UNAIDS (2013) UNAIDS 2013 Report.

3. "http://www.usaid.gov/AAD70867-5C5C-4EB4-A9DD -26D3A9836311 FinalDownload/Download," [Online]. [Accessed 15 October 2014].

4. Federal Ministry of Health (FMOH) Abuja 2010 (2010) Nigeria Integrated Biological and Behavioural Surveillance Survey.

5. Grossman Cl, Stangl AL (2013) Editorial: Global action to reduce HIV stigma and discrimination. J Int AIDS Soc 16: 18881.

6. Logie C, Gadalla TM (2009) Meta-analysis of health and demographic correlates of stigma towards people living with HIV. AIDS Care 21: 742-753.

7. Nyblade L, Stangl A, Weiss E, Ashburn K (2009) Combating HIV stigma in health care settings: what works? J Int AIDS Soc 12: 15

8. Altman D, Aggleton P, Williams M, Kong T, Reddy V, et al. (2012) Men who have sex with men: stigma and discrimination. Lancet 380: 439-445.

9. Fay H, Baral SD, Trapence G, Motimedi F, Umar E, et al. (2011) Stigma, health care access, and HIV knowledge among men who have sex with men in
Malawi, Namibia, and Botswana. AIDS Behaviour 15: 1088-1097.

10. Baral S, Adams D, Lebona J, Kaibe B, Letsie P, et al. (2011) A cross-sectional assessment of population demographics, HIV risks and human rights contexts among men who have sex with men in Lesotho. J Int AIDS Soc 14: 36.

11. Lane T, Mogale T, Struthers H, Mclntyre J, Kegeles SM (2008) They see you as a different thing": the experiences of men who have sex with men with healthcare workers in South African township communities. Sex Transmission Infection 84: 430-433.

12. Rispel LC, Metcalf CA, Cloete A, Moorman J, Reddy V (2011) You become afraid to tell them that you are gay: health service utilization by men who have sex with men in South African cities. J Public Health Policy 32 Suppl 1: S137151.

13. Moses R, Mkuye MS, Magembe GE, Yotham WL, Mellah AO, et al (2012) Barriers to sexual reproductive health services and rights among young people in Mtwara district, Tanzania: a qualitative study. Pan African Medical Journal 13: 13.

14. Van der Elst EM, Smith AD, Gichuru E, Wahome E, Musyoki $H$, et al (2013) Men who have sex with men sensitivity training reduces homoprejudice and increases knowledge among Kenyan healthcare providers in coastal Kenya. Journal of the International AIDS Society 16: 18748.

15. ***http://www.cdc.gov/std/hiv/STDFact-STD-HIV.htm

16. Boyce C, Neale P (2006) Using Mystery Clients: A Guide to Using Mystery Clients for Evaluation Input. Pathfinder International Tool Series.

17. Federal Ministry of Health, Nigeria, National Guidelines on the provision of Syndromic management of STIs and other Reproductive Tract Infections, Abuja: National AIDS and STI Control Programme, 2007.

18. ${ }^{* * *}$ http://www.healthit.gov/providers-professionals/improved-diagnosticspatient-outcomes

19. Keni BH (2006) Training competent and effective Primary Health Care Workers to fill a void in the outer islands health service delivery of the Marshall Islands of Micronesia. Hum Resour Health 4: 27. 